\title{
Application Classifications of Decentralized Finance and the Development Strategies
}

\author{
Xidi Zhang ${ }^{1 *}$ \\ ${ }^{1}$ School of Finance, Zhejiang University of Finance \& Economics, Hangzhou, Zhejiang, 310018, China \\ *Corresponding author. Email: 1848099854@qq.com
}

\begin{abstract}
Decentralized finance is a decentralized network based on smart contracts of blockchain technology and specific decentralized applications (DAPPS). It not only can enhance users' information privacy and transaction equity, but also has incomparable advantages in protecting asset security, reducing financial cost and de-trust. The opportunities of decentralized financial development include: Solving the problem of information asymmetry in traditional financial transactions; Providing safe and convenient asset management and inclusive finance; Promoting the formation of broader global financial services. In order to better promote decentralized finance development, we need to actively follow and embrace its progress trend; Establish "weakly centralized" structure based on Consortium Blockchain; Strengthen international coordination and cooperation of RMB; Improve the regulatory systems and relevant laws and regulations.
\end{abstract}

Keywords: Decentralization Finance, Blockchain

\section{PREFACE}

With the gradual deepening of the blockchain application, blockchain has brought disruptive changes to the financial industry. The upgrade of Ethereum's Layer2 and Eth2 have greatly improved its network performance, paving the way for the evolvement of decentralized finance. Flattening the threshold of financial services through trying to move the traditional financial products to the decentralized network, decentralized finance establishes a multi-faceted, fair and transparent financial service ecosystem to realize inclusive finance, thus opening up a brand new development space in the financial field.

\section{THE CONCEPT AND APPLICATION CLASSIFICATIONS OF DECENTRALIZED FINANCE}

\subsection{The Concept of Decentralized Finance}

Decentralized Finance, or DEFI, is a decentralized network based on smart contracts of blockchain technology and specific decentralized applications (DAPPS). This technology network applies the characteristics of distributed ledger, transparency and censorship resistance in "decentralization" to the traditional financial field, thus establishing an open source code, permissionless, transparent, correlative, and multifaceted system of financial products, reinventing and improving the existing financial system.

The application of decentralized finance changes the asset end, the capital end and the transaction end of original funds, so that the fund transactions between system users do not need to be conducted through thirdparty intermediaries. Based on decentralized financial technology, users carry out and implement peer-to-peer (P2P) interaction with the ecosystem through specific decentralized applications (DAPPS) while maintaining complete control of their assets [1].

\subsection{Application Classification of Decentralized Finance}

Analyzing the thousands of decentralized financerelated applications (DAPPS) that have been exploited on Ethereum, the current types of applet in decentralized finance mainly involve three categories: issuance, transaction and ownership management. The contents cover many aspects such as creating financial assets that can be traded, transferred and meet the needs of users, providing liquidity, swap trading and forecasting market trading, offering asset management and payment network services. 


\subsubsection{The Classification Of Issuance Application And Successful Case}

The first category of application in published DAPPS is issuance, which refers the creation of financial assets that can be traded, transferred and met user needs. It includes three subcategories: stability tokens, lending, and issuance of securities, insurance, and non-homogeneous tokens [2]. They provide the possibility to achieve currency stability, broaden the coverage of financial lending services, and create new financial products and services.

Compound is one of successful examples of lending in published DAPPS. Based on Ethereum, the algorithmic, independent interest rates DEFI protocol is mainly used to realize the business which is similar to the "mortgage lending" of banks. Users can pledge their assets in the agreement to receive an annualized return, while the lender of the asset has to pay the corresponding interest. The interest rates that debits and credits pay and receive are determined by the supply and demand of each crypto asset[3]. Exploiting each block will generate an interest rate, and the user can repay the loan and withdraw the locked assets at any time. At the same time, users can mortgage one token to lend another token, such as mortgage ETH and lend the US dollar stablecoin USDC.

\subsubsection{The Classification Of Transaction Application And Successful Case}

The second category in published DAPPS of applications is trading, which consists of three subcategories: decentralized trading, derivatives and their swap trading and forecasting market trading, as well as liquidity provision, It achieves the goal of immediate custody and breaks withdrawal limits, and better meets the liquidity demand of processing large orders.

Uniswap is one of successful examples of trading. It is a decentralized trading protocol built on the Ethereum blockchain that provides unmanaged transactions for ERC-20 tokens. In Uniswap, users can exchange tokens and add tokens to the pool to earn fees or token trading pairs without trusting any central intermediary. Since all interactions occur directly on the chain, transaction fees are incurred. Uniswap is built on a unique system called Automatic Market Maker (AMM), which at its core is a pool of two ERC-20 tokens to create liquidity. As a reward for the liquidity clause, the party (the liquidity provider or LP) charges each person an interchange fee for exchanging tokens.

\subsubsection{The Classification Of Ownership Management Application And Successful Case}

The third category of application in published DAPPS is ownership management, which includes three subcategories: wallet, asset management and payment network. It realizes the effective storage, transfer and appreciation of assets and improves the speed and efficiency of on-chain and inter-chain payment [4].

OKEx is a success example of assets management. It is one of the world's famous digital asset trading platforms, which mainly offers BTC, LTC, ETC and other digital assets of coin exchange services and derivatives trading services to users around the world. OKEx adopts advanced technologies such as GSLB, distributed server cluster, distributed storage, high-speed memory transaction engine for multiple devices to back up each other, cold wallet, hot wallet with private key offline, etc., to provide customers with secure, stable and reliable digital capital transaction services with multiple terminals such as Web, mobile and PC terminals. At the same time, it continues to improve and upgrade its products and services according to users' suggestions and needs, aiming to provide better and better services to every user.

\section{OPPORTUNITIES AND CHALLENGES OF DECENTRALIZED FINANCIAL DEVELOPMENT}

\subsection{Opportunities of Decentralized Financial Development}

\subsubsection{Solving The Problem Of Information Asymmetry In Traditional Financial Transactions}

In traditional finance, due to the existence of thirdparty intermediaries, customers mainly rely on financial intermediaries to obtain transaction information, which makes it impossible or difficult to conduct direct communication between customers. As a result, the information contents of both parties, including credit, supply and demand, are not perfect and comprehensive, and the cost of information acquisition is high. Based on blockchain technology, decentralized finance provides a new and effective method that connects the two sides of a financial transaction directly to solve information asymmetry between the two parties in traditional financial transactions. So that both the demander and the supplier of services can effectively and conveniently obtain the credit, assets, liabilities and other relevant information of the other party which enriches the transaction details and types. In this way, the optimal effect of financial services can be brought into play and people's financial needs can be met more fully. 


\subsubsection{Providing Safe And Convenient Asset Management And Inclusive Finance}

Centralized finance is controlled, managed and provided with user data by third-party intermediaries. However, because financial institutions cannot ensure the complete accuracy of data records and exclude malicious tampering by others, users are often in a passive state, which brings great troubles. In a decentralized financial system, users maintain complete control of their assets, and secure transactions between users without central authentication can be achieved through decentralized applications (DAPPS), which improve the privacy and security of transactions. Based on the characteristics of immutability and permanent preservation of blockchain, the application of blockchain technology replaces the dependence on centralized institutions with data blocks, and replaces the central or bilateral mutual trust with social or multilateral public trust, making decentralized finance more secure [5].

\subsubsection{Promoting The Formation of Broader Global Financial Services}

Decentralized finance reduces the requirements for user identity, wealth, etc, and lowers the threshold of use and reduces service delivery differences. Everyone can access the financial services he or she needs via the Internet or a smart phone. In the meantime, the use of whole network accounting mode of blockchain makes cross-border bookkeeping possible. It reduces the cost of cross-border credit establishment and international remittance, thus promoting the international financial asset flow, which is conducive to the formation of a broader global financial services.

\subsection{Challenges of Decentralized Financial Development}

\subsubsection{Shocking The Traditional Monetary System And Intensifying The Monetary Supervision Confusion}

In the economies of various countries, central banks, based on national credit, become the monopoly institution of currency issuance, which is also regarded as an important symbol of national exercise of sovereignty. However, the issuance of virtual digital currency based on blockchain technology is not based on national credit. It is a currency beyond sovereignty, without country and supervision. It marks the transformation from centralized state credit to decentralized algorithmic credit, challenges the state's function of issuing currency, and weakens the concept of state and government. Since the central bank cannot influence the macro economy through adjusting monetary policy, this also leads to the increasing instability of money demand and monetary multiplier. In addition, digital currencies may compete with commercial banks on deposits to some extent, and there is an "impossible triangle". It cannot meet the three conditions of high efficiency, decentralization and sufficient security at the same time, which is easy to cause financial disintermediation and panic, thus hitting the traditional monetary system and intensifying the monetary supervision confusion.

\subsubsection{Being Trapped by Strong Technical Limitations And Having Significant Performance Bottlenecks}

At present, decentralized financial applications mainly rely on Ethernet. Ethereum is a Public Blockchain, where all information is public, and there is no way to access control and centralized auditing. Smart contracts have financial features and can store large asset values. These bottlenecks pose great threats to the security of smart contract, which put forward high technical requirements for the performance of the underlying Public Blockchain. In addition, blockchain has small storage capacity and a large number of copies, which makes the situation of low throughput and high latency exist for a long time. Therefore, the scalability of the blockchain system needs to be matched with the volume of transactions to achieve synchronous increase in order to avoid congestion caused by a surge in transactions and the impact on the liquidity of financial assets.

\subsubsection{Insufficient User Cognition Level and Quantity}

Decentralized financial projects put forward higher requirements on user's operational level and knowledge structure. At the current stage, the lack of user awareness results in the small number of users. The "LEGO-style" free combination of decentralized financial applications (DAPPS), on the one hand, enriches the types of financial services. On the other hand, it also increases the difficulty for users to identify financial products and services, making it difficult for users to grasp the financial risks behind them, thus reducing the use of such products. In addition, due to the fact that face-to-face approach can better bridge the digital divide, decentralized financial projects are difficult to establish and accumulate users' trust in some areas where digital methods are not well popularized and with a high degree of population aging, causing users to reject the use of such projects. 


\section{COUNTERMEASURES TO PROMOTE DECENTRALIZED FINANCIAL DEVELOPMENT}

\subsection{Actively Follow And Embrace Decentralized Finance Progress Trend}

The rapid development of decentralized financial technology has become an irresistible trend. The development of technology is bound to bring about disruptive changes in the financial industry. Therefore, the financial industry must take the initiative to change their service concept, technology and process with a positive attitude, so as to achieve new development in the 21st century through innovation. At the same time, following the development of decentralized finance is also an inevitable requirement to better meet the multiple market demand. It not only provides financial services to people in less developed areas, but also enables people in developed areas to experience more transparent and fair finance [6].

\subsection{Establish "Weakly Centralized" Structure Based On Consortium Blockchain}

Consortium Blockchain is a blockchain technology that targets only members of a specific group and limited third parties. A number of pre-selected nodes are designated as bookkeepers internally, and the generation of each block is jointly decided by all the pre-selected nodes, thus forming a "weakly centralized" structure. Users first establish network access rules, which allow them to provide or receive financial services among themselves, thus forming alliances and sharing transaction data. Because the Consortium Blockchain has a strict access mechanism externally and a loose consensus mechanism internally, the structure can also guarantee the security and give consideration to the transaction efficiency. In addition, in order to avoid congestion caused by surging trading volumes, the scalability of the blockchain system needs to match with trading volumes to achieve synchronous increase and avoid having an impact on the liquidity of financial assets.

\subsection{Strengthen International Coordination and Cooperation of $R M B$}

The virtual digital currency based on blockchain technology has broken the traditional centralization of central banks, and the traditional money and banking theories will not be able to fully adapt to the development of practice. Therefore, it is more important to accelerate the theoretical research of digital currency under the new situation. In addition, the central bank should promote the international coordination and cooperation of the RMB, form a benign interaction, and lay the foundation for the international development of the digital RMB. At the same time, the central bank should ensure that the financial sector serves the substantial economy to prevent it from becoming a product of excessive speculation, and focus on protecting internal financial stability and consumer rights and interests.

\subsection{Improve The Regulatory Systems and Relevant Laws and Regulations}

Decentralized finance makes the decentralized system replace the national control of the financial sector, weaken the boundary between finance and technology, and increase the difficulty of responsibility implementation and accountability. Therefore, it is necessary to establish a complete, efficient and rigorous supervision system, further clarify departmental responsibilities and select technical personnel. It is also necessary to strengthen digital financial infrastructure construction and implement hierarchical management. Furthermore, the current laws and regulations related to decentralized finance in China seriously lag behind the social development and are almost in a blank state. The legislation lag will inevitably lead to the lack of regulatory basis, which brings great trouble to the government. Therefore, it is urgent to improve relevant laws and regulations, introduce effective multi-party systems such as privacy protection, and standardize and guide the healthy and sustainable development of decentralized finance.

\section{CONCLUSION}

Decentralized finance can provide financial services to the public in a more efficient, fair and open way, making payments, loans and transactions without permission and censorship restrictions. The data that exists on the Blockchain is transparent, visible and cannot be tampered with. These characteristics of decentralized finance make it possible to solve the problems existing in the traditional financial system, such as the isolation of the existing financial infrastructure, the inefficiency and high cost of international banking and remittance services; Billions of people around the world do not have bank accounts and fair access to financial services. Technological development is a signal of industry change. The traditional financial sector must quickly adapt to the development of decentralized finance, accessing to more favourable interest rates and global liquidity through the protocol system, and relying on technological innovation to achieve more long-term development.

\section{REFERENCES}

[1] Zhao Quanhou, Xu Jing. The Realistic Obstacles of Digital Currency Decentralization and Its 
Implications for Financial Supply Side Reform. Development Finance Research,2019 (5), pp9-18.

[2] Zhang Jinxue. He Yuying, Research on the Difficulty and Countermeasures of Financing Channel of China's Small Loan Companies. Modern Business,2018(1), pp221-222.

[3] Zhu Tao, Zhang Zhiguang. "Decentralization" of Digital Financial Inclusion: A Study Based on the Blockchain Project GSenetwork. China Southern Finance,2019(4), pp63-72.

[4] Yang Tao, Wang Bin. Decentralized finance and blockchain. Financial Expo (Fortune), 2016(6), pp18-19.

[5] Liu Shuangliang. Development and Evolution of Blockchain Finance, Outstanding Difficulty and Promotion Path. Research on Financial Theory,2020(2), pp3-10.

[6] Wei Zuntian. Research and design of financial core trading system based on distributed technology. Electronic Measurement,2020(19), pp78-79. 